\title{
The impact of patient comorbidity on cancer stage at diagnosis
}

\author{
Jason Gurney ${ }^{*},{ }^{1}$ Diana Sarfati ${ }^{1}$ and James Stanley ${ }^{1}$ \\ ${ }^{1}$ Department of Public Health, University of Otago, PO Box 7343, Wellington, New Zealand
}

Background: It is known that cancer stage is affected by comorbidity, but the evidence regarding the magnitude and even direction of this effect is highly inconsistent and poorly understood. The aims of this study were to establish the impact of comorbidity on cancer stage at diagnosis, using both specific individual comorbid conditions and a global measure of comorbidity; and to assess whether this impact varied by cancer site, level of comorbidity burden and individual comorbidity type.

Methods: We examined comorbidity among 14096 patients with breast, colon, rectal, liver, stomach, ovarian, uterine, bladder or kidney cancer. Patients were identified from cancer registry data, and then linked to hospitalisation data to determine the presence of comorbidity in the 5 years preceding cancer diagnosis. Individual comorbid conditions were identified using ICD-10 codes, and overall burden of comorbidity attributed using a cancer-specific measure of comorbidity (C3 Index).

Results: We observed that the presence of patient comorbidity (a) increases the odds of being diagnosed with distant metastases, (b) does not lead to earlier diagnosis and (c) increases the likelihood of a patient receiving no stage of disease at diagnosis.

Conclusions: Patient comorbidity has a substantial impact on cancer stage at diagnosis; however, this impact varies considerably by cancer type, individual comorbid condition and overall comorbidity burden.

It is known that cancer stage at diagnosis is affected by patient comorbidity, but the evidence regarding the magnitude and even direction of this effect is highly inconsistent (Terret et al, 2009; Corkum et al, 2012). This is because there are several competing mechanisms that may impact on stage at diagnosis (Fleming et al, 2005). Increased contact with health services may result in a 'surveillance effect' - leading to earlier diagnosis. In contrast, comorbidity may distract both the clinician and the patient from early signs and symptoms of cancer - leading to delayed diagnosis. In some cases, the patient has such severe comorbidity that their life expectancy is so limited that diagnostic investigation does not appear warranted. Furthermore, some comorbid conditions (e.g., diabetes) may have a direct effect on cancer growth (Giovannucci et al, 2010). The balance of these mechanisms is likely to vary by comorbidity and cancer type, as well as by health system factors.

The aims of this paper were: (a) to establish the impact of comorbidity on cancer stage at diagnosis across a wide range of cancers, using both specific individual comorbid conditions and a global measure of comorbidity; and (b) assess whether this impact varied by cancer site, level of comorbidity burden and individual comorbidity type.

\section{MATERIALS AND METHODS}

Participants. The current study is part of the wider C3 (Cancer, Comorbidity and Care) study, which investigated the impact of patient comorbidity on cancer care and outcomes. The New Zealand Cancer Registry (NZCR) was used to identify patients diagnosed with one of nine cancers (01 January 2006-31 December 2008), which were then clustered into five cancer 'groups': female breast (ICD-10-AM code: C50), colorectal (C18-C20), gynaecological (ovarian (C56) and uterine (C54)), upper gastrointestinal (liver (C22) and stomach (C16)) and urological (bladder (C67) and kidney (C64)). These cancers were included to represent a range of cancers that varied in terms of patient characteristics and underlying burden of comorbidity.

*Correspondence: Dr J Gurney; E-mail: jason.gurney@otago.ac.nz

Received 23 April 2015; revised 7 September 2015; accepted 14 September 2015; published online 13 October 2015 
Patients were excluded if they were diagnosed with carcinomain situ, aged $<25$ years at diagnosis, non-New Zealand residents, had a previous diagnosis of the same cancer or were diagnosed post mortem. Our final cohort included $n=14096$ patients.

Data sources. Cancer Registry data were linked to public hospital (and reporting private hospital) discharge data (National Minimum Data Set (NMDS)) via a unique identifier, for the 5 years before the cancer diagnosis.

Variables. Sex, age at diagnosis, prioritised ethnicity, domicile code, cancer site, date of diagnosis and stage (SEER Summary Stage; categorised as local, regional, distant and unknown (Young et al, 2000)) were determined from the NZCR. For each patient, an index date was defined as the first admission that occurred at or within four weeks of date of cancer diagnosis, with the index cancer as the primary diagnosis. Where no such admission was identifiable, index date was the date of cancer diagnosis on the NZCR.

Level of patient deprivation was determined using the $2006 \mathrm{New}$ Zealand Deprivation Index (NZDep) using domicile data (missing for 5.1\%) (Salmond and Crampton, 2012).

All comorbid conditions recorded on the NMDS in the 5 years before the index hospitalisation date were identified, and used to calculate a C3 Index score for each patient (Sarfati et al, 2014). The C3 Index is a cancer-specific index of comorbidity, which is calculated based on the presence of 42 chronic conditions - each weighted according to its impact on non-cancer mortality among cancer patients, and then summed to arrive at a comorbidity score (Sarfati et al, 2014). C3 Index scores were categorised into ' 0 ' $(\leqslant 0)$, ' 1 ' $(\leqslant 1.00)$, ' 2 ' $(\leqslant 2.00)$ and ' 3 ' $(>2.00)$.
Conditions that might be closely related to the primary cancer of interest or its treatment were excluded (Supplementary Material 5), while conditions that may have been complications of the primary disease or its treatment were only included if they were recorded before the index date (Supplementary Material 6).

Statistical analysis. Analysis was performed in SAS (v9.3, SAS Institute Inc., Cary, NC, USA). Key demographic, disease and comorbidity characteristics were described and standardised to the total New Zealand cancer population. Multinomial logistic regression methods were used to assess the extent to which overall level of patient comorbidity and individual comorbid conditions impacted on stage of disease adjusting for age (continuous), sex (where relevant), cancer group or site (as relevant), ethnicity (Māori/ nonMāori) and deprivation. When the impact of individual conditions was being assessed, we limited adjustment to age, sex, ethnicity and cancer group (for full cohort) because of limited numbers (methodology detailed in Supplementary Material 7).

Ethical approval was gained through the New Zealand Health and Disability Ethics Committee (reference \#: MEC/10/042/EXP).

\section{RESULTS}

Table 1 shows the characteristics of the cohort in relation to their overall comorbidity status, with these data stratified by cancer type in Supplementary Material 3. Those in higher comorbidity categories tended to be older, proportionately less likely to be female, more likely to be Māori and more likely to live in more deprived areas (Table 1).

Table 1. Patient characteristics for the total cohort, stratified by C3 Index category

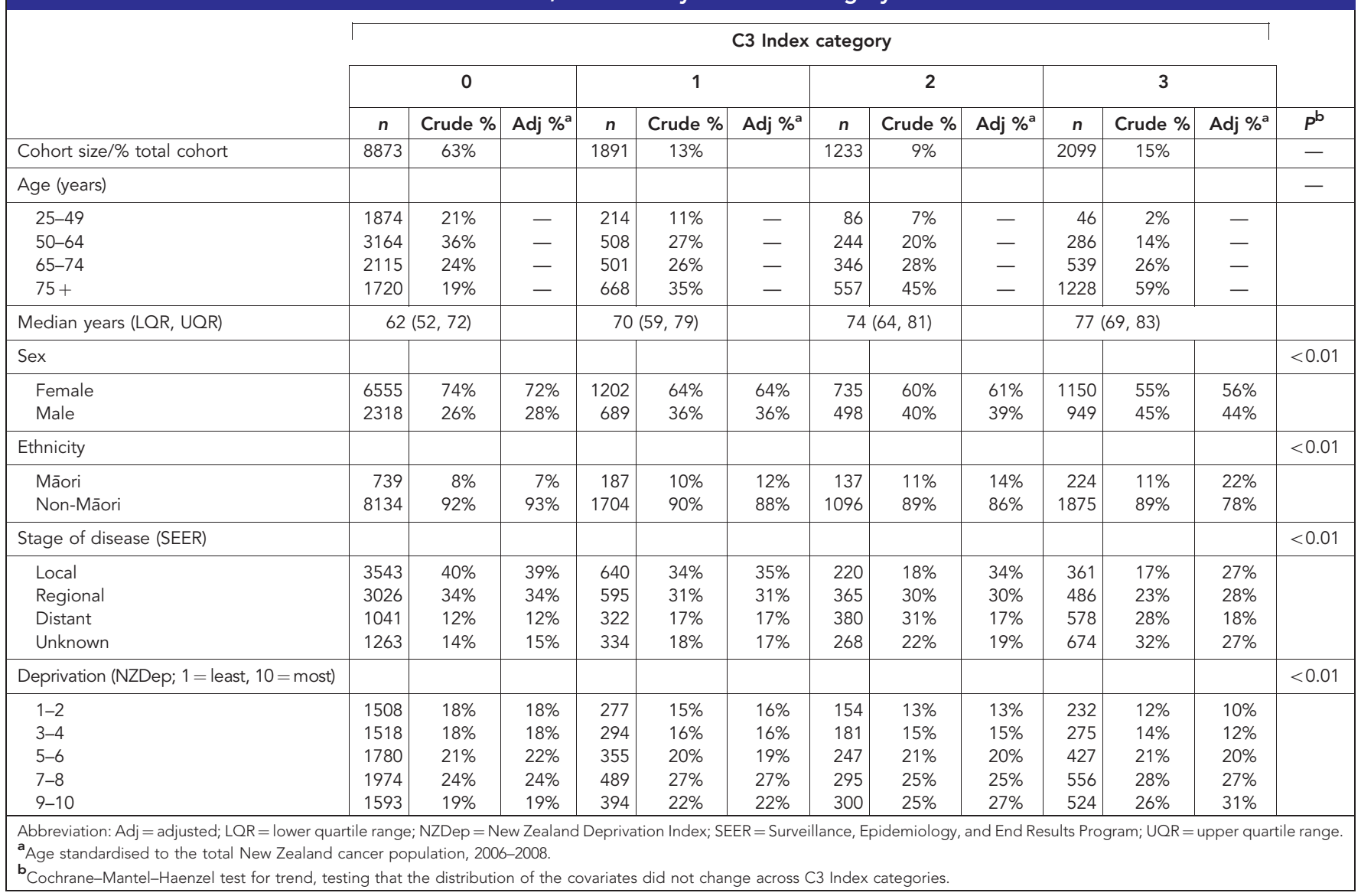


There was substantial variation in overall comorbidity burden between cancer groups. For example, more than a quarter $(26 \%)$ of upper GI patients were observed to have the highest level of overall comorbidity (C3 Index category ' 3 '), compared with only $7 \%$ of the breast cancer cohort (Supplementary Material 1). The crude prevalence of individual comorbid conditions are presented by cancer group in Supplementary Material 2.

The impact of both overall comorbidity burden and individual comorbid condition on stage of disease at diagnosis is presented in Tables 2 and 3. For the total cohort, the odds of having distant disease (rather than local) increased with rising levels of comorbidity, with higher odds of distant stage for those in C3 Index category '2' (adjusted OR: 1.29, 95\% CI 1.06-1.57) and category ' 3 ' (1.49, 95\% CI 1.26-1.77), compared with patients in C3 Index category ' 0 '. The pattern for individual cancer sites were generally similar to those observed for the total cohort, especially for those in the highest comorbidity category; however, estimates were imprecise for gynaecological, urological and upper GI cancers. Regarding unstaged disease, those with the highest comorbidity burden (C3 Index category ' 3 ') had $85 \%$ greater odds of being unstaged at diagnosis compared with those without comorbidity (adjusted OR: 1.85, 95\% CI 1.59-2.16; Tables 2 and 3).

Several comorbid conditions increased the odds of distant disease at diagnosis, with dementia having the strongest individual impact Table 3). Alcohol abuse disorders, neurological conditions and pulmonary circulation disorders resulted in more than a doubling of the odds of distant disease at diagnosis. Several other conditions increased the odds of distant disease at diagnosis by at least $50 \%$, including cerebrovascular disease, congestive heart failure and major psychiatric disorders. Only chronic viral hepatitis and intestinal disorders appeared to be associated with decreased odds of distant disease at diagnosis. In all, 27 of the 42 investigated comorbid conditions were observed to increase the odds of unstaged disease (Table 3; Supplementary Material 4).

\section{DISCUSSION}

This study investigated the degree to which patient comorbidity the presence of chronic conditions other than the primary tumour - might impact on stage at diagnosis. Our observations among 14096 cancer patients suggest that the presence of patient comorbidity (a) increases the odds of a patient being diagnosed with distant metastases, (b) does not lead to earlier diagnosis and (c) increases the likelihood of a patient receiving no stage of disease at diagnosis.

Contrary to the 'surveillance effect', which suggests that increased contact with health services due to the presence of comorbidity may result in earlier diagnosis, this study found no pattern of earlier stage at diagnosis with higher comorbidity levels. This observation is in contrast to those observed in some contexts, where higher comorbidity levels have been associated with earlier

Table 2. Impact of comorbidity burden on stage of disease at diagnosis (adjusted odds ratios (OR) from multinomial logistic regression models), for the total cohort and by cancer group

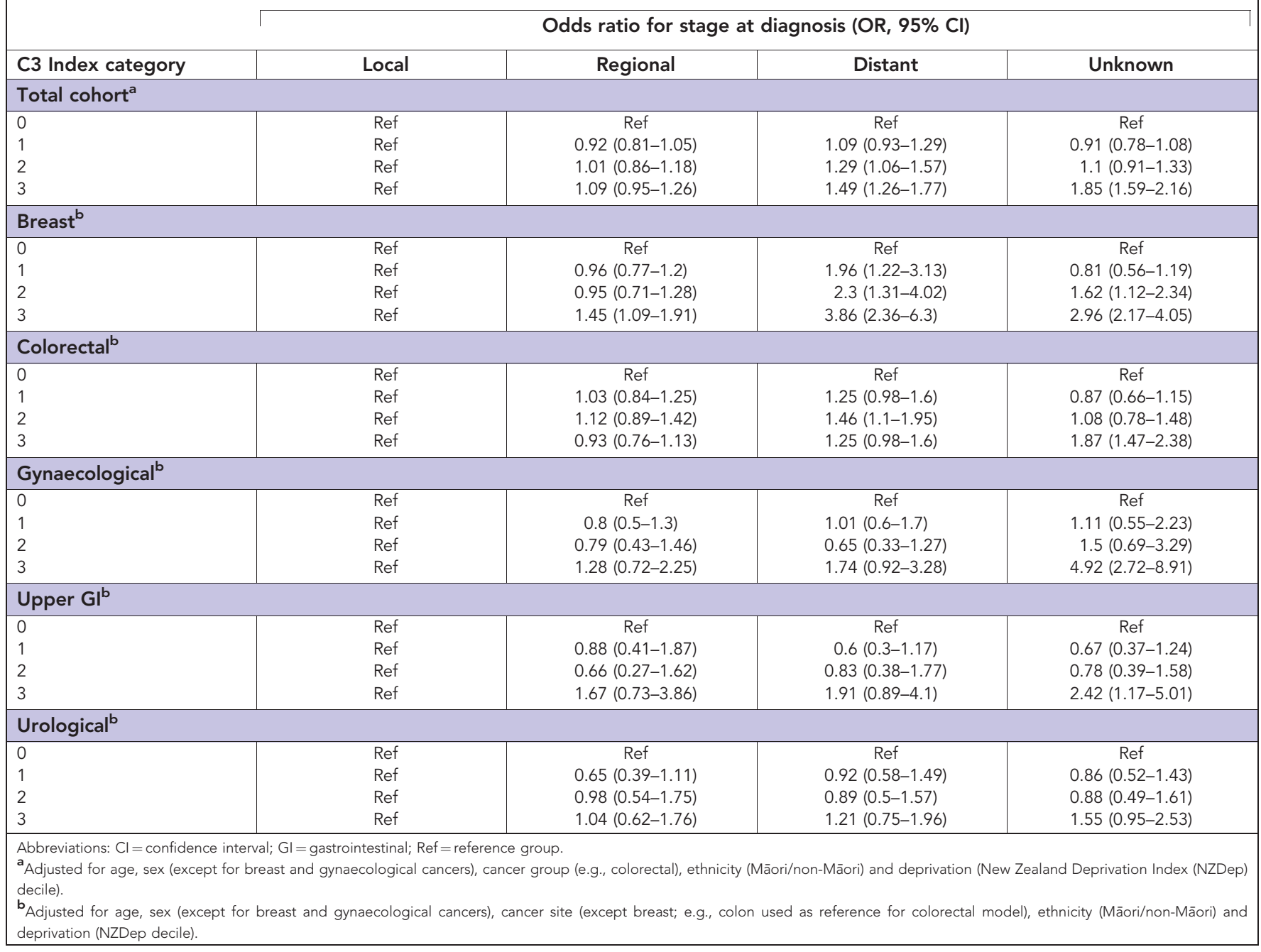


Table 3. Impact of individual comorbidities on stage of disease at diagnosis (adjusted odds ratios (ORs) from multinomial logistic regression models), for the total cohort

Odds ratio for stage at diagnosis $(\mathrm{OR}, 95 \% \mathrm{Cl})$

\begin{tabular}{|c|c|c|c|c|}
\hline Comorbid condition & Local & Regional & Distant & Unknown \\
\hline \multicolumn{5}{|l|}{ Total cohort ${ }^{a}$} \\
\hline Alcohol abuse ('Yes' vs 'No') & Ref & $1.91(1.06-3.46)$ & $2.4(1.25-4.61)$ & $3.6(1.98-6.55)$ \\
\hline Anaemia & Ref & $1(0.8-1.23)$ & $0.94(0.72-1.22)$ & $1.03(0.81-1.32)$ \\
\hline Angina & Ref & $1.01(0.8-1.27)$ & $0.9(0.67-1.21)$ & $1.16(0.9-1.5)$ \\
\hline Anxiety and behavioural disorders & Ref & $0.97(0.61-1.54)$ & $1.45(0.83-2.51)$ & $1.58(0.96-2.58)$ \\
\hline Cardiac arrhythmia & Ref & $0.95(0.79-1.15)$ & $1.17(0.94-1.46)$ & $1.58(1.3-1.91)$ \\
\hline Cardiac valve disorder & Ref & $0.78(0.57-1.08)$ & $1.02(0.7-1.49)$ & $1.43(1.04-1.96)$ \\
\hline Cerebrovascular disease & Ref & $1.02(0.79-1.31)$ & $1.56(1.18-2.07)$ & $1.55(1.2-2.01)$ \\
\hline Congestive heart failure & Ref & $1.11(0.85-1.45)$ & $1.54(1.14-2.07)$ & $2.83(2.21-3.63)$ \\
\hline Coagulopathy/blood disorders & Ref & $1.13(0.94-1.35)$ & $1.29(1.05-1.59)$ & $1.33(1.09-1.62)$ \\
\hline Connective tissue disease & Ref & $0.78(0.46-1.33)$ & $0.87(0.45-1.67)$ & $1.85(1.13-3.03)$ \\
\hline COPD & Ref & $0.87(0.69-1.08)$ & $1.29(1-1.66)$ & $1.74(1.4-2.16)$ \\
\hline Dementia & Ref & $2.33(1.22-4.46)$ & $6.25(3.27-11.96)$ & $8.72(4.81-15.81)$ \\
\hline Diabetes no complications & Ref & $0.9(0.74-1.1)$ & $1.07(0.84-1.35)$ & $0.87(0.69-1.1)$ \\
\hline Diabetes with complications & Ref & $1.24(1-1.55)$ & $1.18(0.91-1.53)$ & $1.31(1.03-1.66)$ \\
\hline Endocrine disorders & Ref & $1.59(1.07-2.35)$ & $1.61(1-2.57)$ & $1.49(0.95-2.33)$ \\
\hline Epilepsy & Ref & $0.95(0.45-1.99)$ & $1.99(0.9-4.37)$ & $1.38(0.6-3.15)$ \\
\hline Eye problems & Ref & $1.24(0.93-1.67)$ & $1.23(0.85-1.76)$ & $1.39(1.01-1.91)$ \\
\hline Gl disease & Ref & $0.97(0.7-1.36)$ & $1.44(1.02-2.03)$ & $1.19(0.85-1.65)$ \\
\hline Hepatitis: chronic viral & Ref & $0.23(0.11-0.45)$ & $0.42(0.24-0.73)$ & $1.47(0.94-2.29)$ \\
\hline Hypertension & Ref & $0.93(0.81-1.07)$ & $1.08(0.92-1.27)$ & $1.34(1.16-1.55)$ \\
\hline Inflammatory bowel disorder & Ref & $1.24(0.99-1.56)$ & $1.4(1.06-1.84)$ & $1.42(1.1-1.85)$ \\
\hline Inner ear disorder & Ref & $0.89(0.61-1.31)$ & $0.97(0.61-1.54)$ & $1.3(0.89-1.91)$ \\
\hline Intestinal disorders & Ref & $0.78(0.65-0.94)$ & $0.55(0.43-0.72)$ & $0.59(0.47-0.75)$ \\
\hline Joint or spinal disorders & Ref & $0.97(0.65-1.45)$ & $1.74(1.13-2.68)$ & $1.65(1.1-2.46)$ \\
\hline Liver_moderate/severe disease & Ref & $0.73(0.48-1.12)$ & $1.21(0.8-1.82)$ & $2.06(1.42-2.99)$ \\
\hline Major psychiatric condition & Ref & $1.14(0.76-1.71)$ & $1.72(1.07-2.76)$ & $1.67(1.06-2.61)$ \\
\hline Malnutrition & Ref & $1.45(0.79-2.65)$ & $1.95(0.99-3.84)$ & $2.6(1.42-4.74)$ \\
\hline Metabolic disorder & Ref & $1.11(0.93-1.32)$ & $1.27(1.04-1.56)$ & $1.07(0.88-1.31)$ \\
\hline Myocardial infarction & Ref & $1.07(0.84-1.37)$ & $1.22(0.92-1.62)$ & $1.78(1.4-2.26)$ \\
\hline Neurological conditions excl. epilepsy & Ref & $1.86(1.19-2.89)$ & $2.26(1.36-3.75)$ & $3.54(2.28-5.49)$ \\
\hline Obesity & Ref & $1.12(0.85-1.46)$ & $0.74(0.52-1.05)$ & 1.09 (0.79-1.51) \\
\hline Osteoporosis and bone disorders & Ref & $0.85(0.49-1.47)$ & $1.71(0.96-3.03)$ & $2.84(1.78-4.56)$ \\
\hline Other cardiac conditions & Ref & $0.97(0.8-1.19)$ & $0.94(0.73-1.21)$ & $1.28(1.04-1.58)$ \\
\hline Other malignancy & Ref & $0.97(0.77-1.23)$ & $1.24(0.96-1.62)$ & $1.2(0.93-1.55)$ \\
\hline Paralysis & Ref & $0.9(0.63-1.3)$ & $1.62(1.1-2.39)$ & $1.54(1.08-2.19)$ \\
\hline Peripheral nerve or muscular disorder & Ref & $1.42(0.78-2.57)$ & $1.61(0.81-3.21)$ & $1.68(0.89-3.19)$ \\
\hline Pulmonary circulation disorder & Ref & $1.09(0.59-2.01)$ & $2.41(1.29-4.48)$ & $2.42(1.33-4.39)$ \\
\hline Peripheral vascular disease & Ref & $1.12(0.81-1.54)$ & $1.43(0.99-2.06)$ & $1.41(1.02-1.96)$ \\
\hline Renal disease & Ref & $1.04(0.8-1.35)$ & $1.3(0.97-1.75)$ & $1.68(1.3-2.17)$ \\
\hline Sleep disorder & Ref & $1(0.55-1.83)$ & $0.79(0.38-1.67)$ & $0.72(0.34-1.53)$ \\
\hline Urinary tract disorder & Ref & $1.35(0.86-2.11)$ & $1.56(0.95-2.55)$ & $2.44(1.61-3.7)$ \\
\hline Venous insufficiency & Ref & - & - & $4.07(1.62-10.22)$ \\
\hline
\end{tabular}

stage at diagnosis (Vaeth et al, 2000; Gross et al, 2006; Zafar et al, 2008; Ahn et al, 2013). This pattern has most commonly been reported for screen-detected cancers (breast and colorectal), supporting the contention that in some instances a higher number of visits to health clinics may be related to higher rates of screening - particularly where screening coverage rates are related to health service funding or quality indicators, which may encourage the screening of those with high levels of comorbidity (Fisher et al, 2005; Walter et al, 2009). In the New Zealand context, we found no evidence of this.

By contrast, some of our findings support the so-called 'competing demands' hypothesis, which suggests that the presence of comorbidity can distract patients and/or clinicians to the extent that the early symptoms of tumour growth may go unnoticed (Fleming et al, 2005). For example, we observed that breast cancer patients with the highest overall burden of comorbidity had nearly four times greater odds of being diagnosed with distant metastases than those with no comorbidity burden. These findings are consistent with those of several other studies relating to multiple cancer types (Gonzalez et al, 2001; Miller et al, 2003; Koppie et al, 2008; Tetsche et al, 2008; Teppo and Alho, 2009; Sarfati et al, 2011; Grann et al, 2013).

Some studies have shown that more severe (or 'unstable') comorbid conditions are associated with poorer stage at diagnosis, whilst less severe comorbid conditions are associated with earlier diagnosis (Fleming et al, 2005; Yasmeen et al, 2011). Our own observations support the former, but not the latter - out of the 42 individual comorbid conditions (all of which were included due to their association with non-cancer death in a cancer population; Sarfati et al, 2014) a total of 15 conditions showed increased odds of a patient being diagnosed with distant metastases (OR ranging between 1.27 and 6.25). A further 11 conditions showed similar but non-statistically significant results. By contrast, only two comorbid conditions (hepatitis and intestinal disorders) decreased the likelihood of being diagnosed with advanced disease $(\mathrm{OR}=0.42$ and 0.55 , respectively). It is not possible to speculate from the data whether these two exceptions do indeed represent a surveillance effect. 
The presence of non-cancerous chronic conditions provides a clinical opportunity for earlier cancer diagnosis and referral for efficacious (and evidence-based) screening. Our observations suggest that it is possible that in some instances we may be missing this opportunity.

We also found strong evidence that the presence of comorbidity, particularly a high overall burden, makes it less likely that a patient will be recorded as staged at diagnosis. This observation is consistent with earlier work (Gurney et al, 2013). A possible explanation for this association is that the clinician may decide not to put a patient with severe comorbidity through diagnostic investigation, particularly where that investigation may place the patient at high risk of complication and/or the patient has poor prognosis (as is the case in upper GI cancer, for example). This is, however, speculative.

A major strength of this study is the high-quality nature of the national-level data used. However, there are inherent weaknesses with using administrative data to identify comorbidity. Data may be missing or inaccurate; it can be difficult to differentiate complications of disease from pre-existing conditions; and there may be biases inherent in coding practices. These errors are likely to be non-differential in relation to stage at diagnosis, and are unlikely to account for the associations seen. It is also possible that there may be some differential measurement error, for example, those with later-stage of a given cancer may have been be more likely to have been hospitalised in the period before their diagnosis, resulting in a higher likelihood of their comorbid conditions being recorded. However, we do not believe that this is likely to be a strong effect and thus would be unlikely to have a substantial impact on the general patterns of associations observed here.

The comorbidity status of those treated solely in non-reporting private hospitals may be underestimated, which may result in some bias if there is an association of use of these hospitals with stage of disease at diagnosis. Because we use a 5 -year look back period and because for many cancers use of private hospitals for treatment is unusual in New Zealand, we do not think this bias is likely to explain the results. However for cancers for which private hospital care is more common (such as breast and colorectal), we cannot exclude the possibility that the association of lower comorbidity with earlier stage at diagnosis may, at least in part, be explained by this effect.

As we did not correct for multiple comparisons in our analysis of the independent role of each of the 42 comorbid conditions on stage at diagnosis, individual confidence intervals for these conditions should be interpreted with caution; however, we have observed a clear pattern of association across conditions. Finally, it should be noted that the low prevalence of some individual comorbid conditions is likely to have affected our power to detect significant differences for this component of the study.

\section{CONCLUSIONS}

We observed that patient comorbidity (a) increases the odds of a patient being diagnosed with distant metastases, (b) does not lead to earlier diagnosis and (c) increases the likelihood of a patient receiving no stage of disease at diagnosis. The strength of these associations varies by cancer type, individual comorbid condition and overall comorbidity burden.

\section{ACKNOWLEDGEMENTS}

We thank the wider C3 (Cancer, Comorbidity and Care) research team, and the Health Research Council of New Zealand for funding this study (HRC ref \#: 10/496). This study was funded by the Health Research Council of New Zealand.

\section{CONFLICT OF INTEREST}

The authors declare no conflict of interest.

\section{NOVELTY AND IMPACT OF PAPER}

This retrospective cohort study showed that comorbidity among cancer patients (a) increased the odds of being diagnosed with distant metastases, (b) did not lead to earlier diagnosis and (c) increased the likelihood of a patient receiving no stage of disease at diagnosis.

\section{REFERENCES}

Ahn DH, Mehta N, Yorio JT, Xie Y, Yan J, Gerber DE (2013) Influence of medical comorbidities on the presentation and outcomes of stage I-III non-small-cell lung cancer. Clin Lung Cancer 14(6): 644-650.

Corkum M, Urquhart R, Kendell C, Burge F, Porter G, Johnston G (2012) Impact of comorbidity and healthcare utilization on colorectal cancer stage at diagnosis: literature review. Cancer Causes Control 23: 213-220.

Fisher DA, Judd L, Sanford NS (2005) Inappropriate colorectal cancer screening: findings and implications. Am J Gastroenterol 100(11): 2526-2530.

Fleming ST, Pursley HG, Newman B, Pavlov D, Chen K (2005) Comorbidity as a predictor of stage of illness for patients with breast cancer. Med Care 43(2): 132-140.

Giovannucci E, Harlan DM, Archer MC, Bergenstal RM, Gapstur SM, Habel LA, Pollak M, Regensteiner JG, Yee D (2010) Diabetes and cancer: a consensus report. Diabetes Care 33(7): 1674-1685.

Gonzalez EC, Ferrante JM, Van Durme DJ, Pal N, Roetzheim RG (2001) Comorbid illness and the early detection of cancer. South Med J 94(9): 913-920.

Grann A, Froslev T, Olesen A, Schmidt H, Lash TL (2013) The impact of comorbidity and stage on prognosis of Danish melanoma patients, 1987-2009: a registry-based cohort study. Br J Cancer 109: 265-271.

Gross CP, Andersen MS, Krumholz HM, McAvay GJ, Proctor D, Tinetti ME (2006) Relation between Medicare screening reimbursement and stage at diagnosis for older patients with colon cancer. JAMA 296(23): 2815-2822.

Gurney J, Sarfati D, Stanley J, Dennett E, Johnson C, Koea J, Simpson A, Studd R (2013) Unstaged cancer in a population-based registry: prevalence, predictors and patient prognosis. Cancer Epidemiol 37(4): 498-504.

Koppie TM, Serio AM, Vickers AJ, Vora K, Dalbagni G, Donat SM, Herr HW, Bochner BH (2008) Age-adjusted Charlson comorbidity score is associated with treatment decisions and clinical outcomes for patients undergoing radical cystectomy for bladder cancer. Cancer 112(11): 2384-2392.

Miller DC, Taub DA, Dunn RL, Montie JE, Wei JT (2003) The impact of co-morbid disease on cancer control and survival following radical cystectomy. J Urol 169(1): 105-109.

Salmond C, Crampton P (2012) Development of New Zealand's Deprivation Index (NZDep) and its uptake as a national policy tool. Can J Public Health 103(S2): S7-S11.

Sarfati D, Gurney J, Stanley J, Salmond C, Crampton P, Dennett E, Koea J, Pearce N (2014) Cancer-specific administrative data-based comorbidity indices provided valid alternative to Charlson and NHI indices. J Clin Epidemiol 67: 586-595.

Sarfati D, Tan L, Blakely T, Pearce N (2011) Comorbidity among patients with colon cancer in New Zealand. N Z Med J 124(1338): 76-88.

Teppo H, Alho O-P (2009) Comorbidity and diagnostic delay in cancer of the larynx, tongue and pharynx. Oral Oncol 45(8): 692-695.

Terret C, Castel-Kremer E, Albrand G, Droz JP (2009) Effects of comorbidity on screening and early diagnosis of cancer in elderly people. Lancet Oncol 10(1): $80-87$. 
Tetsche MS, Dethlefsen C, Pedersen L, Sorensen HT, Norgaard M (2008) The impact of comorbidity and stage on ovarian cancer mortality: a nationwide Danish cohort study. BMC Cancer 8: 31.

Vaeth PA, Satariano WA, Ragland DR (2000) Limiting comorbid conditions and breast cancer stage at diagnosis. J Gerontol A Biol Sci Med Sci 55(10): M593-M600.

Walter LC, Lindquist K, Nugent S, Schult T, Lee SJ, Casadei MA, Partin MR (2009) Impact of age and comorbidity on colorectal cancer screening among older veterans. Ann Intern Med 150(7): 465-473.
Yasmeen S, Xing G, Morris C, Chlebowski RT, Romano PS (2011) Comorbidities and mammography use interact to explain racial/ethnic disparities in breast cancer stage at diagnosis. Cancer 117(14): 3252-3261.

Young J, Roffers F, Gloeckler Ries L, Fritz A (eds) (2000) SEER Summary Staging Manual-2000: Codes and Coding Instructions. National Cancer Institute: Bethesda, MD, USA.

Zafar SY, Abernethy AP, Abbott DH, Grambow SC, Marcello JE, Herndon 2nd JE, Rowe KL, Kolimaga JT, Zullig LL, Patwardhan MB, Provenzale DT (2008) Comorbidity, age, race and stage at diagnosis in colorectal cancer: a retrospective, parallel analysis of two health systems. BMC Cancer 8: 345 .

Supplementary Information accompanies this paper on British Journal of Cancer website (http://www.nature.com/bjc) 